\title{
CURSOS COMUNITARIOS: una primera alternativa para el medio rural
}

Elsie Rockwell ${ }^{86}$

Hace veinte años, y como único en su género, se desarrolló el sistema de Cursos Comunitarios en México. El sistema ofrece educación primaria a niños que habitan comunidades rurales donde aún no existen escuelas regulares. Su programa combina los aciertos el sistema formal con las ventajas del no-formal. Es de cobertura nacional, y financiado y administrado por el gobierno; no obstante, también promueve una intensa participación comunitaria. El programa es equivalente al nacional y ofrece el certificado oficial de primaria; funciona, sin embargo, mediante jóvenes semiprofesionales que usan materiales diseñados específicamente para el trabajo multigrado. Cursos Comunitarios está integrado al sistema educativo nacional; aún así, ha desarrollado modos flexibles de organización y de enseñanza multigrado, con objeto de ofrecer con mayor eficacia la primaria completa.

Cursos Comunitarios fue establecido en 1973 por el Consejo Nacional de Fomento Educativo Conafe, una agencia gubernamental y descentralizada que maneja varios programas educativos innovadores. En 1975, y posteriormente en 1989, el Conafe solicitó a varios investigadores educativos del Centro de Investigación y de Estudios Avanzados del Instituto Politécnico Nacional (CINVESTAV-IPN) DE México que desarrollan el currículo y los materiales para Cursos Comunitarios. En el presente trabajo se examinarán algunos aspectos del desarrollo del modelo, se analizará el diseño experimental del currículo y los materiales, y se discutirán los criterios necesarios para evaluar la eficiencia y calidad de Cursos Comunitarios.

\section{Llevar la escuela a los niños, no los niños a la escuela}

Las innovaciones educativas deben, con frecuencia, su impulso inicial a momentos políticos particularmente intensos. Cursos Comunitarios no fue una excepción, pues nació en la década de los 70, cuando el gobierno mexicano tenía las razones y los recursos suficientes para proyectar una reforma a gran escala. A pesar de que ha sido cuestionada en años recientes, la Reforma Educativa de los 70 logró un considerable avance cualitativo y cuantitativo de la educación en el país.

Un desafió enfrentó México durante los 70, garantizar el acceso universal a las escuelas primarías para cubrir la totalidad de la "demanda efectiva", lo que excluía a ciertos grupos, entre ellos, a la "población dispersa" en aldeas aisladas, donde no se consideraba rentable establecer una escuela oficial. Bajo el impulso de la reforma, la Secretaria de Educación Pública (SEP) buscó alternativas para proporcionar educación primaria a los niños de miles de comunidades con menos de 100 habitantes.

Se propusieron varias soluciones. Algunas autoridades sugirieron revivir el programa de "escuelas de circuito", con maestros ambulantes. Otros favorecían el sistema de internados establecidos para niños indígenas rurales. También se consideraba apropiado dotar a las comunidades con fondos federales para contratar maestros locales. Sin

${ }^{86}$ Elsie Rockwell (Mexico). Antropóloga educativa. Investigadora en el Centro de Investigación y de Estudios Avanzados del Instituto Politécnico Nacional (IPN) en México. Ha coordinado varios proyectos de desarrollo curricular, entre ellos "Dialogar y Descubrir", para el programa Cursos Comunitarios. 
embargo, la logística de estas propuestas resultaba poco práctica, y el Sindicato Nacional de Trabajadores de la Educación se opuso a la existencia de un sistema educativo paralelo.

Surgieron, entonces, dos soluciones, de características contrastantes: los albergues indígenas y los Cursos Comunitarios. En las regiones indígenas, la SEP contrataba jóvenes bilingües, como promotores, para enseñar castellano, lectura y escritura en los primeros grados de primaria completa a los niños indígenas de las comunidades dispersas; se establecieron albergues en los pueblos, donde los alumnos viven de lunes a viernes para poder asistir a las escuelas de estas poblaciones.

Para los niños rurales no-indígenas, la SEP puso en marcha, a través del Consejo Nacional de Fomento Educativo (Conafe), el sistema de Cursos Comunitarios. Inicialmente, los instructores comunitarios del programa impartían sólo los primeros dos o tres grados. Sin embargo, en muchas localidades los padres llegaron a exigir que se ampliara el servicio para cubrir los grados superiores. Cuando las evaluaciones comparativas mostraron que en los cursos se lograban resultados equivalentes a los de las primarías cercanas, se decidió que los instructores ofrecieran la primaria completa (seis grados). Gracias a este sistema fue posible llevar la escuela a los niños, evitando así los costos y riesgos que implicaba el envío de los niños hasta las escuelas de los pueblos.

\section{La recuperación de tradiciones educativas}

Al diseñar Cursos Comunitarios se retomaron ciertas tradiciones educativas mexicanas, un hecho que se debería tomar en cuenta cuando se consideren programas similares en otros países.

Las Escuelas Rurales establecidas por el gobierno post-revolucionario en México durante los años 20 y 30 ofrecían precedentes importantes. La revolución (1910-1917) había generado una demanda significativa de escuelas públicas en las áreas rurales. La población del medio rural financiaba, voluntariamente, la construcción y el equipamiento de esas escuelas y vigilaba el trabajo escolar. Durante aquellos años, los maestros rurales eran semiprofesionales; ingresaban al servicio sin formación normalista y recibían cursos de capacitación en servicio, así como frecuentes visitas de sus supervisores. Habitualmente, trabajaban con tres o cuatro grados al mismo tiempo. Uno de sus logros fue conseguir que se aceptara la coeducación en el medio rural durante una época en que las escuelas urbanas aún separaban a niños y niños. Tras el impulso de las reformas en los años, 20, estos maestros consideraban la acción social y el desarrollo comunitario como parte integral de su trabajo. A pesar de que las escuelas rurales fueron incorporadas gradualmente al modelo general de la escuela urbana, sus logros sobrevivieron en la memoria de los educadores y la gente del medio rural a lo largo del país. De esta manera, contribuyeron a ilegitimizar el programa de Cursos Comunitarios.

Otros antecedentes también incidieron en el diseño de los Cursos Comunitarios. En México, todos aquellos que reciben educación superior deben proporcionar un servicio social para obtener el grado o certificado de estudios. Este fue un precedente importante para establecer el sistema de becas que el Conafe ofrece a los jóvenes que realizan un servicio como instructores comunitarios.

El diseño de un currículo para Cursos Comunitarios también consideró algunas disposiciones nacionales. A lo largo del presente siglo 
se ha generalizado una convicción igualitaria en la educación mexicana: se ha sostenido que la educación primaria debe dar a todos los niños acceso al mismo conocimiento básico, y que ningún sector recibirá una educación "de segunda". En una de las acciones encaminadas a favorecer esta igualdad, el gobierno mexicano desarrolla y distribuye, desde 1960, libros de texto gratuitos nacionales de cada materia para todos los niños que asisten a la primaria en el país. Estos libros de texto fueron el recurso pedagógico y la referencia básicos en el desarrollo del currículo para el programa de Cursos Comunitarios.

\section{Una estructura alternativa para ofrecer la primaria}

Para lograr una educación universal se requiere establecer estructuras flexibles, alternativas, dentro de un sistema integrado. La larga búsqueda de un modelo claro y funcional para Cursos Comunitarios ilustra este punto. Desde un principio, el sistema se planeó para funcionar a escala nacional. Inicialmente cubrió 100 localidades en un estado, y alcanzó los 10.000 cursos en su totalidad de los 31 estados en cinco años. Inicialmente, Cursos Comunitarios fue concebido como una forma de promover y subsidiar "escuelas privadas" mediante una transferencia de recursos a comités locales, que se encargarían entonces de contratar a los instructores. Como el Conafe asumió, cada vez más, la administración y el desarrollo del sistema, la modalidad inicial se volvió insostenible. Sin embargo, la institución no podía establecer legalmente un sistema paralelo de contratación de maestros.

En 1980 se desarrolló el modelo actual, que combina servicio social y becas a los instructores. Ingresan al programa, como instructores comunitarios, jóvenes originarios del medio rural, de edades entre 15 y 21 y que hayan completado la escuela secundaria, (grados 7 a 9). Al principio, se matriculaban paralelamente en un sistema especial, "abierto", para completar dos años de estudio post—secundarios mientras realizaban su trabajo como instructores. Para finalizar el tercer año dejaban el servicio y asistían a clases regulares, recibiendo la misma compensación. Esta doble responsabilidad afectó tanto a sus estudios como a su actividad docente. Por ello, el modelo fue modificado. Ahora, los instructores trabajan uno o, si lo desean, dos años en una comunidad, recibiendo alimentos, habitación, y un pago mensual aproximadamente equivalente a US\$100 ${ }^{87}$. Al terminar este período, los exínstructores reciben una beca por tres años (US\$90 mensual) para proseguir su propia educación en la escuela que elijan.

El carácter institucional del Consejo Nacional de Fomento Educativo garantizó la viabilidad del Programa. El Conafe depende directamente de la Secretaria de Educación Pública (SEP); no obstante, tiene personería jurídica y presupuesto independientes, y funciona fuera de la estructura administrativa que se encarga de la educación primaría. Los funcionarios del más alto nivel son designados por el Secretario, pero un equipo técnico relativamente estable se ha hecho cargo durante largo tiempo del desarrollo de Cursos Comunitarios, así como de otros programas de educación comunitaria (servicio pre-escolar rural, educación para la salud, publicaciones, programas de becas). La oficina central establece las pautas de administración y de capacitación, publica materiales didácticos y complementarios, y elabora los exámenes empleados para la evaluación y la acreditación. El Conafe opera por medio de Delegaciones estatales que cuentan con un reducido personal técnico y administrativo. Este se encarga de la capacitación, la supervisión y la evaluación para los programas educativos.

${ }^{87}$ El salario mínimo legal en México es ligeramente más alto, y los maestros de nuevo ingreso ganan aproximadamente US450. 
Los instructores comunitarios reciben, por lo general, un curso intensivo de dos meses durante el verano que precede a su ingreso en el servicio. El personal a cargo de los cursos de capacitación ha sido de diversos tipos a lo largo de la existencia del programa: han participado desde maestros con experiencia en escuelas rurales hasta jóvenes con estudios universitarios. Sin embargo, el sistema ha retornado una y otra vez a lo que parece ser la mejor opción: hoy en día, la capacitación es impartida principalmente por ex-instructores, quienes permanecen dentro del sistema por uno o dos años adicionales. Además de dar el curso básico, estos capacitadores trabajan durante el año escolar como "tutores", con diez a veinte instructores a su cargo. Visitan las comunidades y coordinan reuniones mensuales, donde los instructores cobran su compensación, intercambian experiencias, discuten problemas comunes y revisan materiales. Los capacitadores, a su vez, reciben cursos anuales y son supervisados por el personal técnico de Conafe.

La participación de la comunidad es un componente básico del sistema. Las localidades a las que llega el servicio de Conafe albergan más o menos a una decena de familias. Los habitantes eligen un comité local rotatorio denominado Asociación Promotora de Educación Comunitaria (Apec). Las Apec firman anualmente un contrato con Conafe, donde aceptan alojar y mantener al instructor y adecuar o construir un local para la escuela. Establecen el horario para el curso y promueven la inscripción de niños y jóvenes, sin limite de edad o escolaridad previa. Los representantes de la Apee se reúnen con el personal de Conafe tres veces al año y proporcionan constantes informes sobre la marcha de los cursos.

Los instructores permanecen en la comunidad la mayor parte del tiempo. Por ello, establecen vínculos estrechos con los padres de familia y pueden discutir con ellos el progreso de cada niño o niña. Los padres, a su vez, pueden supervisar el trabajo del instructor, sugerir soluciones prácticas a determinados problemas y, por lo general, influir en el trabajo escolar. Al principio se impartían clases en alguna de las casas; actualmente casi todas las comunidades atendidas cuentan con un local escolar construido a veces con la ayuda financiera de los gobiernos locales. Frecuentemente, los instructores se involucran en otras actividades comunitarias, promueven campañas de salud o de alfabetización de adultos, y ofrecen ayuda para proyectos de desarrollo. Así, establecen fuertes lazos entre la comunidad y los programas de Conafe.

\section{Dialogar y descubrir: Aprovechando las ventajas de la escuela unitaria}

El modelo pedagógico y los materiales didácticos para el programa Cursos Comunitarios fueron desarrollados por un equipo de investigadores del Departamento de Investigaciones Educativas del Centro de Investigación y de Estudios Avanzados del Instituto Politécnico Nacional (DIE-CINVESTAV-IPN), mediante convenio con el Consejo Nacional de Fomento Educativo. Los primeros manuales del instructor comunitario se habían elaborado por el DIE entre 1975 y 1978 y se utilizaron durante quince años. Considerando los cambios que se habían hecho en los programas nacionales y los libros de texto, en 1988 el Conafe solicitó al DIE que renovara los materiales. El equipo del DIE elaboró una nueva serie de 9 libros, titulada Dialogar y descubrir, que han sido editados entre 1989 y 1993. Se utiliza el material completo por primera vez durante el año escolar 1993 - 1994. En ambos casos, participaron miembros del personal técnico de Conafe y un grupo de instructores comunitarios, particularmente en el proceso de experimentación del material en el campo. La metodología y la conceptualización pedagógica desarrolladas por el equipo que elaboró la serie Dialogar $y$ descubrir han sido contribuciones significativas al campo educativo. 
Un desafío importante en la elaboración de un currículo para Cursos Comunitarios fue el de lograr que los contenidos fueran equivalentes a los del currículo nacional y, además, relevantes para la población de las localidades atendidas. Quedaba claro, a través del trabajo d campo, que un currículo relevante no tenía que ser, necesariamente un currículo "rural". Los niños del medio rural necesitan y desean aprender muchas cosas que no forman parte de su entorno inmediato; los temas de realidades distantes o pasadas pueden resultar fascinantes para ellos. Además, existen actividades —como los juegos, las ilustraciones y los experimentos- que poseen un atractivo intrínseco para todos los niños. Por otra parte, no se propuso que la primera debe proveer habilidades e información susceptibles de adquirirse más eficientemente por otros medios, como la participación directa en el trabajo, en la vida social o en los proyectos comunitarios. Así, los contenidos de Cursos Comunitarios pueden parecer más convencionales que aquellos frecuentemente incorporados a programas de educación no-formal. Sin embargo, los contextos extraescolares y el conocimiento cultural local brindaron, de hecho, referentes fundamentales al seleccionar y presentar un conocimiento escolar significativo para los niños.

Ya que el sistema escolar mexicano pretende garantizar la igualdad y permitir la movilidad en todo el sistema educativo, fue importante asegurar la equivalencia del currículo de Cursos Comunitarios con el programa nacional. Sin embargo, era necesario introducir algunos cambios. En la selección de los contenidos, el equipo procuró, más que conservar una paridad formal con los programas oficiales, establecer una equivalencia real con aquello que de hecho se enseña en la primaria y, a la vez, promover un mejor aprovechamiento. Basándose en investigaciones recientes, el equipo consideró que algunos de los temas oficiales de cada grupo se planteaban en los programas vigentes en 1988 de una manera demasiado compleja o extensa para su eficaz enseñanza en la primaría, independientemente del medio social. Por ello, se eliminaron aquellos temas que se consideraron inadecuados para cualquier niño-y no sólo para el medio rural- en determinado grado. Muchos de estos cambios habían sido sugeridos también por otros educadores (la reducción de los temas de gramática, por ejemplo) y han sido subsecuentemente incorporados a la actual reforma educativa de primaria en México. Al reducir contenidos y enfatizar más bien el enfoque pedagógico, el programa de Dialogar y descubrir espera promover un aprendizaje más significativo y duradero de los conceptos y las competencias esenciales.

En cierto sentido, el principal tema no fue definir el contenido temático, sino readecuar la estructura del currículo oficial. Cuando los programas para cada grado marcan una secuencia diferente de temas y materias, el maestro de escuela unitaria completa tiene que trabajar con seis temas diferentes al mismo tiempo, lo cual es muy difícil de lograr. En realidad, la enseñanza multigrado sólo es factible si el docente puede enseñar temas similares simultáneamente a varios grados. Esta condición requiere el diseño de un "currículo cíclico", es decir, una programación en la cual por lo menos algunos contenidos se repitan en los sucesivos grados o esos contenidos cada año y comparten los conocimientos que ya han adquirido con alumnos de grados inferiores. El equipo que trabajó en Dialogar y descubrir consideró que esta estructura cíclica ofrecía ventajas, pues los niños podrían abordar los conceptos básicos varias veces, desde distintos niveles de desarrollo, y así ampliar su comprensión y competencia a medida que progresaran en la primaria.

Siguiendo esta lógica, el equipo reordenó los contenidos para los seis grados en tres niveles, que corresponden a los "ciclos" de dos grados, y se cursan normalmente en dos 
años cada uno. Así, al trabajar con toda la primaria, los instructores organizan actividades para sólo tres subgrupos, en lugar de seis. Cada materia -Español, Matemáticas, Ciencias Naturales, Historia y Geografía - comprende cuatro o cinco unidades por nivel. Los temas que eran considerados para un grado en los programas oficiales se hicieron extensivos a todo el nivel o bien se eliminaron. Muchos temas, incluso, coinciden para dos niveles, aunque se distinguen actividades para cada nivel. Las materias complementarias —educación física, artística y tecnológica- se trataron colectivamente, y fueron integradas a las actividades propuestas en otras. Esta estructura guió la elaboración de los Manuales del instructor Comunitario, como material básico del programa.

El diseño del currículo tomó en cuenta otras consideraciones. El cálculo del tiempo de clase disponible en un año escolar impuso restricciones a la estructura y al contenido curricular. Las decisiones acerca de qué incluir en cada nivel se basaron en diversos criterios pedagógicos, así como en la relevancia local de cada tema. La selección final de los contenidos no se hizo sino hasta después de un largo proceso de experimentación: toda propuesta fue sometida a la prueba de los niños e instructores en el campo. De esta manera fue posible centrar los materiales de Dialogar y Descubrir en los componentes esenciales del currículo de primaria.

\section{Vincular aprendizaje y enseñanza}

El desarrollo del modelo pedagógico y de los materiales Dialogar y Descubrir se basó en los resultados de investigaciones, realizadas a lo largo de diez años por los miembros del equipo, sobre el aprendizaje y la enseñanza de los contenidos académicos en primaria, así como sobre la vida cotidiana en las escuelas primarias.

Un principio básico es compartido por los autores: los niños del medio rural aprenden fundamentalmente de la misma manera que cualquier niño, aun cuando sus mundos materiales y culturales sean muy diferentes. Las oportunidades de aprender que tienen estos niños pueden estar condicionadas por los recursos de su medio; asimismo, sus maneras de expresarse y relacionarse con el conocimiento están arraigadas en la cultura local. Sin embargo, los procesos básicos que utilizan los niños para darle sentido al mundo son universales. Los niños de cualquier parte construyen su propio conocimiento de los significados y regularidades que encuentran en su ambiente para poder apropiarse el conocimiento que los adultos poseen acerca del mundo. Este proceso no se da en un vacío: requiere de una intensa interacción social de los niños con sus semejantes y con otras personas que posean conocimientos diversos. Los términos dialogar y descubrir expresan este concepto de aprendizaje, que subyace al modelo pedagógico de cursos comunitarios.

Una segunda convicción del equipo se refería a la necesidad de tomar en cuenta los requerimientos de la práctica docente, así como las condiciones materiales de las escuelas, al diseñar las actividades de aprendizaje. Dentro de cualquier escuela, un solo adulto trabaja con un grupo de niños dentro del reducido espacio del aula. Esto impone la organización de un trabajo colectivo, antes que la atención individual a cada niño. Aunque las actividades en equipo y los diversos medios didácticos pueden ampliar las oportunidades de aprendizaje, consideramos que el maestro es, finalmente, irreemplazable. Su tiempo es escaso y debe usarse sabiamente, en estrecha interacción con los niños.

Al diseñar actividades para cursos comunitarios, los autores tuvieron que considerar las condiciones particulares de la escuela unitaria, donde un solo docente debe trabajar 
con niños de distintas edades y capacidades. Enseñar en esta situación a menudo se considera "antipedagógico" 0 , incluso, "imposible". Los investigadores optaron por transformar esta condición en una ventaja. La estructura de escuela unitaria nos obliga a diseñar actividades que mantuvieran el interés de los niños, incluso durante varios años sucesivos y que, por otro lado, promovieran la interacción entre estudiantes de distintos niveles. El hecho de que los instructores no podían atender a todos los niños al mismo tiempo favoreció la adopción de actividades en las cuales los alumnos aprenden de manera más autónoma. El reto fue diseñar materiales o actividades que propiciaran cierta interacción entre los alumnos y, frecuentemente, con otras personas de la comunidad.

Al elaborar los materiales, los autores tuvimos que considerar la compleja relación entre dos procesos: el aprendizaje y la enseñanza. Las actividades diseñadas debían relacionarse con los mundos particulares, culturales y materiales, de los niños. Al mismo tiempo, debían ser factibles dentro de las condiciones de la escuela unitaria. Se distribuyó cuidadosamente el tiempo total de enseñanza en la programación anual de las actividades incluidas en los manuales. Estos materiales prevén que mientras un nivel trabaje con el instructor, los otros dispongan de diversas actividades que puedan realizar con poca atención del instructor. Se consideraron también los intereses, recursos y conocimientos de los instructores al diseñar las actividades.

Para garantizar una mayor correspondencia entre las condiciones reales de Cursos Comunitarios y los componentes pedagógicos del programa, el equipo estableció una metodología para diseñar y probar todos los materiales con unos 30 instructores en servicio. Además, se adoptaron los siguientes criterios para guiar el trabajo, basados en investigaciones previas y en la experiencia de los miembros del equipo:

- Relacionar las actividades de enseñanza con el contenido. Esto implica dejar que los niños se involucren con los contenidos, a través de actividades que les sean significativas, antes de que el docente enseñe procedimientos y conclusiones "correctas". Es importante vincular las actividades de una clase a otra y dar tiempo para que puedan revisarse y, en caso necesario, volver a realizarse.

- Incorporar el conocimiento local al conocimiento escolar. Aun cuando se relacionen con los contenidos escolares, las actividades deben ser abiertas a fin de integrar el conocimiento previo de los niños aprovechar los recursos culturales de la comunidad.

- Diseñar actividades teniendo en mente la enseñanza multigrado. Sugerir diversos niveles de participación en las actividades colectivas, así como formas de enriquecer los materiales para los estudiantes más avanzados. Equilibrar las actividades que dependen del maestro con aquellas que promueven la autonomía de los alumnos.

- Enfatizar la interacción, no la individualización. El trabajo colectivo conduce a un aprendizaje más efectivo. Los niños deben aprender juntos; deben resolver y revisar ejercicios conjuntamente, en grupos de dos a cuatro. Los estudiantes mayores pueden enseñar a los menores.

- Proporcionar materiales accesibles, de calidad, y significativos para los niños. Usar recursos locales, pero también enriquecer el ambiente alfabetizado de la escuela y la comunidad con materiales impresos y escritos. Combinar las actividades en las que se manejen los materiales con otras en que se analicen ilustraciones y textos. Dejar que los niños manipulen y usen el equipo normal de la escuela: libros, pizarrón y materiales didácticos. 
- Incorporar tanto tradiciones como innovaciones pedagógicas. Muchas actividades provechosas (la dramatización, por ejemplo) cuentan con amplia legitimidad al interior de las escuelas, y pueden ser incorporadas más fácilmente que otras propuestas más radicales. No es posible descalificar a toda la enseñanza "tradicional"; por otro lado, muchas actividades "innovadoras" resultan poco practicas en el aula.

- Diseñar sesiones de evaluación periódicas (en lugar de aplicar pruebas constantes), en las cuales los alumnos se expresen tanto oralmente como por escrito. Estas sesiones deben incluir preguntas y revisiones colectivas y proporcionar retroalimentación inmediata. Es importante preparar a los estudiantes para los exámenes oficiales, pero no favorecer que se enseñe únicamente en función del examen.

- Determinar la factibilidad de las actividades propuestas. Lograr que todas se adecuen a:

* las condiciones materiales de la escuela unitaria

* los limites estrictos del tiempo disponible en un año escolar

* las características de un texto que se pueda leer fácilmente.

Estos otros criterios ayudaron a lograr cierto equilibrio en los materiales pedagógicos dirigidos a los Cursos Comunitarios. No todas las actividades propuestas satisfacían cada criterio, por supuesto, pues, incluso, el período de cuatro años de desarrollo resultó demasiado corto para resolver los múltiples problemas encontrados en el trabajo de campo. Pero la experimentación en el campo proporcionó información sobre el desarrollo de las actividades y los resultados inmediatos en el aula. Los miembros del equipo hacían seguimientos de las actividades que realizaban los instructores a partir de los materiales provisionales en las comunidades seleccionadas. Además, observaban cómo los niños e instructores interpretaban los textos y realizaban su trabajo y luego analizaban las propuestas y los trabajos del grupo. Muchas actividades se eliminaron o rediseñaron tras la experiencia en el campo. Todas fueron reescritas muchas veces a fin de garantizar claridad, tanto para los niños como para los instructores.

Los materiales resultantes de la serie Dialogar y descubrir-un texto introductorio, dos manuales para instructores, cuatro cuadernos de trabajo para los alumnos del nivel II, una serie de tarjetas de trabajo para el nivel II y un libro de juegos- tienen otro componente esencial: se insistió en contar con ilustración e impresión de buena calidad. El uso de fotografías blanco y negro y dibujos a línea producidos específicamente para el proyecto tenía una función importante, pues aumentó en gran medida la comunicación efectiva con los instructores y los estudiantes.

Frecuentemente se considera que la investigación y el desarrollo experimental de materiales didácticos es un lujo en países -incluido México- donde el pulso político determina los plazos para las reformas educativas. Rara vez se canalizan recursos hacia la producción de respuestas innovadoras a los constantes problemas relacionados con la universalización de la educación básica. Considero, más bien, que este tipo de desarrollo de alternativas es una necesidad insoslayable para la concreción de los objetivos de la iniciativa de Educación para todos, firmada en la reunión mundial en Jomtien, en 1990. 
Los maestros son el factor más importante de cualquier programa educativo, y las recomendaciones internacionales enfatizan correctamente la formación de docentes y la mejoría de condiciones de trabajo. No obstante, han sido, en gran medida, maestros semiprofesionales quienes han logrado extender el servicio de educación primaria a los sectores más marginados en todo el mundo. En el sistema escolar mexicano, muchos docentes aún no cuentan con estudios de nivel superior. Por otro lado, una elevada tasa de deserción docente ha obligado a los gobiernos estatales a contratar un número creciente de maestros no certificados. Dada esta situación, es interesante analizar la experiencia del Conafe con los instructores comunitarios.

Se han señalado varias desventajas en el hecho de trabajar con maestros semiprofesionales. Los instructores poseen un conocimiento limitado de los conceptos básicos que deben enseñar, pues la enseñanza secundaría —en México y en todas partes - tiende a transmitir un conocimiento especializado antes de que los estudiantes dominen las competencias básicas. El hecho de que los instructores estén en servicio sólo por uno o dos años no permite que acumulen la experiencia necesaria para solventar la carencia de una formación de más alto nivel. Además, muchos ven a los instructores como demasiado jóvenes para cumplir con los múltiples papeles sociales que $s$ esperan de un maestro en el México rural, o, incluso, para ganarse e respeto de alumnos que son casi de su misma edad. Algunas de es tas desventajas se reflejan en la elevada tasa de deserción de instructores y en la dificultad de encontrar jóvenes calificados, problemas que han sido recurrentes en el sistema de Cursos Comunitarios.

Contra todo ello, la mayoría de los instructores llegaron a se maestros efectivos en las comunidades, y a la larga, mejores estudiantes. Los parámetros de madurez en las áreas rurales difieren de los urbanos, y los instructores asumen responsabilidades que van más allá de sus edades. A pesar de que trabajan bajo la vigilancia de los padres de familia, muchos se convierten en líderes en las comunidades; enseñan, y al mismo tiempo aprenden, prácticas que contribuyen a una vida mejor.

Por otro lado, los instructores tienen ciertas ventajas por el hecho de ser jóvenes. Suelen establecer una relación cercana, afectiva, con los niños y se ganan su confianza, ayudándolos así a permanecer en la escuela y a aprender. Frecuentemente son más flexibles que los maestros mayores, y más receptivos a las prácticas educativas innovadoras. Aquellos que disfrutan su trabajo participan en las actividades -los juegos, los experimentos y los proyectos- que se diseñaron para los niños, y aprenden junto a ellos. Por su cercanía a los niños, en edad y experiencia, pueden comprender sus perspectivas y comunicarse más efectivamente con ellos.

Por lo general, los instructores valoran el hecho de que los manuales didácticos de la serie Dialogar y descubrir estén cuidadosamente estructurados, aunque otras personas han cuestionado este aspecto de los materiales. No obstante, contar con los manuales, los instructores trabajan autónomamente $\mathrm{y}$, a menudo, responden en primer término a los padres de familia. Los más creativos utilizan los materiales a la vez que enriquecen o varían las actividades con sus propias ideas. Quienes no los usan, en cambio, tienden a enseñar sólo los contenidos tradicionales, y de manera rutinaria.

Por las mismas razones, los mejores capacitados han sido ex-instructores. A pesar de su juventud, son ellos quienes pueden comunicar con mayor eficacia a los instructores que recién ingresan lo que significa la experiencia en la comunidad. Además, habiendo 
utilizado los materiales, están en una mejor posición para enseñar su uso que quienes, aun siendo profesionales, nunca han manejado un grupo multigrado en el medio rural. La participación de algunos instructores en el desarrollo y la experimentación de los materiales resultó ser fundamental. Con la misma intención, se incorporaron testimonios de instructores al manual de capacitación, La experiencia de ser instructor, desarrollado para el programa.

\section{Eficiencia y calidad}

Es claro que toda alternativa para ofrecer la primaria debería satisfacer ciertos criterios de calidad y eficiencia. Aunque el sistema de Cursos Comunitarios ha recibido tanto elogios como críticas en México, no se ha realizado una evaluación externa integral del programa. Existe información parcial disponible que permite apreciar sus resultados. Sin embargo, lo importante en este momento es abrir la discusión acerca de los criterios utilizados para evaluar el tipo de programas innovadores que presenta Cursos Comunitarios.

\section{Aprovechamiento e impacto}

El modelo pedagógico de Cursos Comunitarios, Dialogar y descubrir pretende impulsar una mejor enseñanza y un aprendizaje significativo y duradero del conocimiento básico, mediante el uso de materiales de alta calidad. Los resultados de este objetivo son particularmente difíciles de mediar cuando se trata de un programa que funciona a escala nacional. Asimismo, resulta complicado establecer el impacto social que el sistema puede tener en las comunidades, así come sobre los instructores y su futuro desempeño en la sociedad.

Las dos únicas evaluaciones comparativas entre el sistema del Conafe y otras escuelas rurales se realizaron en 1978 y $1979^{88}$. Ambos estudios concluyeron que, aunque el nivel general fue bajo, el rendimiento en los Cursos Comunitarios era equivalente, refiriéndose a cuarto grado, al de las escuelas primarías de las mismas regiones. El tercer grado llegó a ser, incluso, superior. Sobre estas bases. se agregó al programa el tercer nivel, correspondiente al quinto y sexto grados. Sin embargo, no se ha hecho un estudio comparativo al nivel terminal de la primaria ${ }^{89}$. El análisis de los exámenes anuales que ha realizado el personal de Conafe muestra que el aprovechamiento, tal como se mide en estas pruebas, sigue siendo relativamente bajo.

Aunque es evidente la necesidad de realizar una nueva evaluación comparativa, quisiera expresar diversas reservas al respecto. La primera atañe el tipo de prueba utilizado, pues los formatos de opción múltiple no logran medir adecuadamente las competencias básicas que pretenden desarrollar —incluyendo, por ejemplo, la capacidad de expresarse por escrito- los programas innovadores, como Dialogar y descubrir. Será importante desarrollar métodos alternativos de evaluación.

En segundo lugar, no es posible atribuir el aprovechamiento escolar a una propuesta pedagógica si no se cumplen varios requisitos. Primero, debe haber transcurrido el tiempo suficiente para que la propuesta se transforme en parte normal de un sistema y se apoye mediante procesos adecuados de capacitación y de evaluación. Por otro lado, es

${ }^{88}$ La primera se hizo en 1978, y fue financiada por la Fundación Ford; véase Suárez, Aurora y Elsie Rockwell, 'Evaluación de Cursos Comunitarios", Informe Final. La otra fue realizada por la Secretaría de Educación Pública, a través de la Dirección de Evaluación, en 1979. Los resultados no fueron publicados.

${ }^{89}$ La Dirección de Evaluación cuenta con nuevos datos comparativos, que parecen confirmar los resultados de los años sesenta. 
necesario contar con un diseño de investigación sofisticado, que integre y controle las variables de capacitación, supervisión y uso real de los materiales. La observación cualitativa de clases debe ser una parte integral de la valoración de este tipo de proyectos. Asimismo, es necesario incorporar a la evaluación general de Cursos Comunitarios la interacción entre el programa y las condiciones o los cambios en las comunidades.

\section{Retención y eficiencia terminal}

Cursos Comunitarios se estableció con el objeto de ofrecer una primaria completa, en una época en que la mayor parte de las escuelas ubicadas en pueblos rurales sólo ofrecía de dos a cuatro grados. Por tanto, la evaluación de la eficiencia terminal del programa de adoptar como parámetro los índices de retención de las escuelas rurales, y no los índices nacionales o los derivados de las escuelas urbanas completas.

Se presentan varios problemas al intentar calcular los índices de retención en el sistema de Cursos Comunitarios. Las estadísticas que publica la Secretaría de Educación Pública para Cursos Comunitarios no son adecuadas, ya que no toman en cuenta su estructura propia de niveles del sistema. A pesar de que el Conafe ha obtenido información sistemática durante los últimos años, no hay manera aún de hacer el seguimiento de generaciones de alumnos. La tasa oficial de deserción es alta (15\%) pero ésta incluye a los niños cuyos cursos fueron suspendidos porque los instructores desertaron o por -que las comunidades se retiraron del programa. Estas cifras también consideran a alumnos cuyas familias emigran, o que se trasladan a otras escuelas. Si se contaran sólo aquellos niños que dejan de asistir al curso, a veces temporalmente, aun cuando sigan viviendo en la comunidad, el índice de deserción anual quizá sería del 3\%. La mayoría de estos niños reporta la necesidad de trabajar como su razón para abandonar el curso.

La matrícula promedio de los últimos cinco años ha sido de aproximadamente 100.000 alumnos, de 6 a 15 años, 5.000 niños, en promedio, han obtenido el certificado de primaria cada año durante este período. Alrededor de tres cuartas partes de los niños matriculados toman el examen final en cada nivel, pues las políticas varían de estado a estado, y no siempre se pide a todos los alumnos que lo presenten. Actualmente, aprueban cerca de la mitad de quienes silo hacen; algunos se promueven después de cursar sólo un año en el nivel. La promoción más alta se da en el nivel II, la más baja en el III.

La matrícula de fin de año en 1991-1992 fue de 82.715. De estos alumnos, el 53\% estaba en nivel I, $27 \%$ en el II, y $20 \%$ en el III. Durante ese año escolar, 5.647 estudiantes recibieron el certificado de primaria, lo que representa casi el $7 \%$ del total de alumnos en los tres niveles. El promedio nacional de certificación sobre la matrícula final total en primaría para el mismo año fue del $13 \%$ y casi del $7 \%$ en las escuelas indígenas bilingües.

Estos datos indican que los Cursos Comunitarios han ampliado de hecho las oportunidades de completar la educación primaria para niños y niñas que se hallan entre los más marginados del país. No obstante, todavía falta mucho para garantizar que un mayor número de niños alcancen el nivel III y completen su educación primaria.

\section{Costo-beneficio}

Los criterios para determinar la relación costo-beneficio varían mucho, y deben establecerse con cuidado al hacer comparaciones entre sistemas. Aparentemente, si el presupuesto total del sistema de Cursos Comunitarios se divide entre la matrícula total, el 
costo por alumno resulta más alto que aquel reportado para las escuelas regulares. Esto ha influido en decisiones encaminadas a reducir el número de comunidades atendidas durante los últimos años. Pero este cálculo incluye componentes no considerados en el sistema regular, tales como las becas de tres años que reciben los ex-instructores (actualmente cerca de 20.000) para continuar sus propios estudios. Conafe ha calculado los costos directos del programa en aproximadamente US\$100 por año por alumno, lo cual es la mitad del costo de su propio programa alternativo, Fiducar, que cubre gastos de transporte y alimentos para niños de comunidades rurales que asiste a primarias completas en pueblos cercanos.

En general, la experiencia de Cursos Comunitarios confirma hecho de que no es posible proporcionar el servicio de primaria a 1 niños de regiones aisladas mediante programas $v$ compensatorios menor costo que el de las escuelas regulares. Las alternativas efectivas requieren mayores recursos, particularmente durante la etapa de desarrollo. Sin embargo, pueden ser comparativamente rentable siempre y cuando se integren a un sistema unificado.

\section{Sostenimiento a largo plazo}

Cursos Comunitarios ha perdurado por más de veinte años, sobreviviendo a varios cambios políticos y administrativos. Nació como u programa de transición, bajo el supuesto de que la "población dispersa" dejaría eventualmente de existir. A pesar de que éste no $\mathrm{h}$ sido el caso, los recortes presupuéstales, las prioridades cambiante y los problemas administrativos han reducido el apoyo gubernamental a Cursos Comunitarios. Hoy, el sistema funciona en aproximadamente la mitad de las comunidades que alguna vez fueron atendidas.

A pesar de los recortes, diversos factores han contribuido a la longevidad de Cursos Comunitarios. La demanda y el apoyo de las comunidades han sido fundamentales en muchos casos. Sin embargo, este apoyo debe ser renegociado cada año, y se pierde cuando los problemas administrativos minan la continuidad del servicio y la confianza en el sistema. Por otra parte, algunas comunidades son incapaces de cubrir los gastos que suponen mantener al instructor y proveer el local escolar. Otras optan por enviar a los niños a escuelas cercanas, sobre todo si éstas ofrecen cierta calidad y mayor continuidad.

La garantía de un presupuesto estatal anual ha contribuido a mantener el programa, como lo ha hecho también el trabajo de integrantes del personal técnico que consideran valioso el sistema de Cursos Comunitarios. La calidad de la propuesta curricular también explica la longevidad del programa. El desarrollo y la modificación de los modelos operativo y pedagógico han respondido a necesidades reales del sistema. Estos factores han asegurado una continuidad mayor a la que suele encontrarse, ya que durante la pasada década fueron suspendidos varios programas innovadores gubernamentales.

\section{Gestión administrativa y técnica}

Los programas que operan a escala nacional, como Cursos Comunitarios, se enfrentan con numerosos problemas administrativos entre ellos la relación entre la gestión centralizada y las iniciativas locales. En el caso de Cursos Comunitarios, la experiencia y la administración del equipo central resultaron esenciales para impulsar la innovación y fijar normas de calidad. El Conafe frecuentemente ha incorporado al personal de las Delegaciones estatales a los procesos de toma de decisiones. También ha apoyado iniciativas locales, otorgando a los equipos de las Delegaciones un margen de decisión en 
los procedimientos de supervisión, capacitación y gestión. Además, algunos integrantes del equipo central tienen años de experiencia en Delegaciones y comprenden la perspectiva regional.

La Secretaria de Educación Pública ha descentralizado recientemente todo el sistema escolar básico, transfiriendo a los estados la administración de las escuelas antes controladas por el gobierno federal. Sin embargo, dispuso que el Conafe mantuviera bajo su gestión los programas educativos comunitarios, con objeto de canalizar fondos especiales hacia las zonas más necesitadas. No obstante, ha sido difícil desarrollar fórmulas efectivas para coordinar la iniciativa local y la dirección central.

Si los equipos locales no cuentan con personal competente, las iniciativas locales, en lugar de impulsar innovaciones, simplemente reproducen las prácticas del sistema escolar tradicional. Los equipos locales del Conafe varían mucho en cuanto a su nivel de compromiso y calidad. El cambio constante de personal y la carencia de programas de formación han afectado la capacidad en las Delegaciones. Desde el principio se cuidó que el funcionamiento de Cursos Comunitarios no dependiera de un personal especializado. No obstante, la participación de los equipos técnicos resulta esencial para el éxito del programa en la práctica.

Aunque Conafe es una dependencia relativamente pequeña, no ha sido inmune a la burocratización. Esta ha provocado un divorcio entre las decisiones técnicas y las administrativas, y genera un papeleo excesivo, que ocupa tiempo e impide la flexibilidad local. También se ha creado problemas debido a los múltiples programas dirigidos a las comunidades, pues los instructores tienen que llenar constantemente largos cuestionarios, llevar a cabo campañas de diferentes tipos y, generalmente, fungir como intermediarios entre la comunidad y un gran número de programas gubernamentales de desarrollo. Aunque estos programas resultan valiosos por sí mismos, sobrecargan el tiempo de los instructores y reducen la atención a los niños que cursan la primaria.

\section{La proyección a escala nacional}

Cursos Comunitarios fue diseñado desde el inicio para proyectarse a escala nacional. En 1981 alcanzó un punto máximo de casi 15.000 cursos, con unos 300.000 estudiantes. Actualmente, sin embargo, llega a menos de 8.000 comunidades. Además, el tamaño de cada grupo ha disminuido, pues, tras varios años de operación del sistema, la edad promedio de los estudiantes en cada comunidad se normalizó. Por otro lado, se establecieron escuelas regulares en las comunidades más grandes, y éstas dejaron de ser atendidas por Conafe.

Aunque Cursos Comunitarios es un programa de escala importante, aporta sólo una pequeña parte de la matrícula total de la educación primaria, sin embargo, se trata en realidad de micro-escuelas que promedian una docena de estudiantes cada una. Así, los niños que asisten a Cursos Comunitarios son solamente una fracción (.6\%) de la matrícula total.

El programa podría extenderse a unos 200.000 niños más, que habitan unas 20.000 comunidades donde el programa seria viable ${ }^{90}$. Sin embargo, esta cifra difícilmente convencerá a los encargados de la planeación nacional de realizar la inversión necesaria.

\footnotetext{
${ }^{90}$ Aunque el censo de 1990 enlista unas 65.000 comunidades no-indígenas con menos de 100 habitantes cada una, muchas de éstas se encuentran cercanas a poblados con escuela, o bien son demasiado, pequeñas para poder sostener al instructor.
} 
Actualmente la "población dispersa" quizá plantea un problema menor, en México, que el de otros grandes grupos que aún carecen de educación primaria completa: todos los niños del campo egresados de las escuelas rurales "incompletas" y que no pueden financiar los gastos de asistir a la escuela más próxima; los hijos de varios millones de trabajadores migrantes temporales que viajan a lo largo del país y a Estados Unidos; decenas de miles de niños indígenas que no asisten a las escuelas albergues para completar la primaria; y un número creciente de desertores en las zonas marginales de las ciudades, en lo que hoy es un país predominantemente urbano.

No es posible cubrir esta demanda mayor mediante la reproducción mecánica de un solo modelo, se requiere el desarrollo de alternativas para enfrentar cada problema especifico. Sin embargo, es posible aprovechar la experiencia e inversión previas del sistema de Cursos Comunitarios para atender a otros niños que carecen de servicios educativos. Así, el Conafe actualmente está probando variantes de este programa en unos 100 campos de trabajadores migrantes. De cualquier forma, la experiencia de Cursos Comunitarios debería extenderse más allá del Conafe, y aprovecharse en el sistema educativo regular, donde podría ayudar a resolver los problemas de enseñanza multigrado en el medio rural. Las propuestas incluidas en los materiales Dialogar y descubrir se han difundido, hasta ahora, sólo de manera informal, mediante el contacto entre los instructores y maestros de otras escuelas.

\section{El vinculo con el sistema nacional}

Uno de los principales retos del programa Educación para Todos acordado en Jomtien en 1990 es el de "crear un sistema integrado", en el que las diversas modalidades se complementen y se refuercen mutuamente. Las posibilidades y la flexibilidad de cualquier sistema alternativo están condicionadas por los vínculos específicos que éste guarde con el sistema nacional.

Cursos Comunitarios se vincula al sistema nacional, principalmente por medio de los instructores, quienes continúan sus estudios en cualquiera de las veinte modalidades de la educación post-secundaria (grados 10 a 12) existentes en el país. Como los exinstructores regresan al sistema regular, los calendarios anuales para Cursos Comunitarios deben coincidir con aquellos establecidos a nivel nacional. Sin embargo, las autoridades locales tienen cierto margen para delimitar el período de vacaciones y reponer clases perdidas.

La acreditación de los estudios también toma en cuenta al sistema nacional. Los padres de familia requieren boletas oficiales al final de cada año, pues los niños deben estar en posibilidad de trasladarse a cualquier otra escuela. Las boletas han suscitado problemas por el pasaje entre una organización flexible de tres niveles y el sistema escolar organizado por grados. Están basadas en el criterio propio que utiliza el Conafe para agrupar y evaluar a los niños, y además, dan una equivalencia con los grados para facilitar, en caso necesario, la transición a otras escuelas. Sin embargo, esto confunde frecuentemente a los padres, y debilita el modelo pedagógico al introducir una referencia a los grados en un sistema organizado por niveles.

Los padres de familia ven a Cursos Comunitarios como una opción legítima porque ofrece un certificado de primaria idéntico al extendido por las escuelas regulares. Sin embargo, como el sistema funciona sin maestros certificados, la SEP ha exigido que los alumnos del programa, a diferencia de sus contrapartes de otras escuelas, presenten un examen nacional estandarizado. En este sentido, para completar la primaria en Cursos Comunitarios, los niños enfrentan criterios más estrictos que aquellos de las escuelas regulares. 
Muchos de los problemas de vinculación se generan porque la estructura educativa general ofrece poca flexibilidad para la organización de alternativas para ofrecer la primaria. A pesar del discurso de la igualdad, no todas las escuelas primarías son iguales. Alrededor de un tercio de ellas tienen maestros que deben trabajar simultáneamente con entre dos y seis grados, siempre cumpliendo con los mismos requisitos impuestos a las escuelas completas, que cuentan con uno o más maestros por grado. Así, los maestros de escuelas multigrado deben cubrir los programas y llenar la documentación de cada grado por separado. La negativa a reconocer que existen estructuras escolares diferentes afecta las condiciones de trabajo y la eficiencia de todas las escuelas en el medio rural, no sólo las de Cursos Comunitarios. No es sorprendente que ambos sistemas enfrenten problemas similares: altos índices de deserción docente, papeleo excesivo que recorta el tiempo dedicado a la enseñanza, y una crisis de legitimidad social.

La politización de las reformas educativas ha causado cambios rápidos y constantes, y la reforma actual en México ha dejado poco tiempo para la consolidación y evaluación de propuestas pedagógicas. Menos aún ha dado condiciones para tomar en cuenta la diversidad del sistema y para permitir cierta flexibilidad en la organización escolar y curricular. La posibilidad de contribuir a los objetivos acordados en Jomtien, mediante esquemas educativos alternativos, se vincula estrechamente con el funcionamiento general del sistema educativo. Los objetivos sólo podrán alcanzarse cuando los sistemas nacionales consideren seriamente la diversidad de problemas involucrados en garantizar una educación universal y de calidad a una población diferenciada.

\section{Bibliografía}

Consejo Nacional de Fomento Educativo, Conafe, Educación Comunitaria, México, Conafe, 1982.

Consejo Nacional de Fomento Educativo, Dirección de Programas y Desarrollo Educativo, Resultados del Aprovechamiento Escolar del Ciclo 1990-1991, México (mimeo).

Consejo Nacional de Fomento Educativo, Dirección de Desarrollo de Sistemas, Compendio de Indicadores Educativos (Ciclo Escolar 1991-1992), México (mimeo).

Fuenlabrada Irma and Eva Taboada, Curriculum and educational research; a innovation for basic education. Prospects No. 81, Paris, 1992.

Suárez, Aurora y Rockwell, Elsie. Consejo Nacional de Fomento Educativo, México, 1978.

Rockwell, Elsie, Candela, Antonia, Fuenlabrada, Irma, Block, David, Navarro, Laura y Taboada, Eva. Investigación básica e innovación didáctica: el nuevo Manual del Instructor Comunitario (Dialogar y descubrir), en: Memorias del Primer Encuentro General de Innovaciones en Educación Básica, Centro de Estudios Educativos Editorial Esfinge, México, 1991.

Secretaria de Educación Pública, Dirección General de Planeación, Programación y Presupuesto, Estadística básica del sistema educativo nacional. Fin de cursos 19911992, México, 1993.

Dialogar y descubrir (Serie para Cursos Comunitarios).

Manual del Instructor Comunitario, Niveles 1 y II, con Fichas. Roekwell, E. 
(coordinadora), D. Block, H. Balbuena, A. Candela, C. Diaz, I.

Fuenlabrada, J. González, L. Navarro, F. Reyes, P. Safa. E. Taboada y S.

Vernon. México, Consejo Nacional de Fomento Educativo (Conafe) y

Departamento de Investigaciones Educativas (DIE) del Centro de Investigación y de Estudios Avanzados del Instituto Politécnico Nacional, 1989.

La experiencia de ser instructor. Roekwell, E. y R. Mercado. Conafe-DIE, México, 1990.

Manual del Instructor Comunitario. Nivel III. Rockwell, E. (coord.), D. Block, A. Candela, I. Fuenlabrada, L. Navarro, E. Taboada, H. Balbuena, J. González, M.C. Larios, G. Quintero, F. Reyes, Conafe-DIE, México, 1992.

Matemáticas, Cuaderno de Trabajo del Nivel III. Block, D., I. Fuenlabrada y H. Balbuena, Conafe-DIE, México, 1992.

Historia y Geografía. Cuaderno de Trabajo de Nivel III. Taboada, E. y F. Reyes, ConafeDIE, México, 1992.

Español. Cuaderno de Trabajo del Nivel III. Navarro, L., G. Quintero y M.C. Larios, Conafe-DIE, México, 1993.

Ciencias Naturales. Cuaderno de Trabajo del Nivel III. Candela, A. y J. González, ConafeDIE, México, 1993.

Libro de Juegos. Niveles I, II, III. Rockwell, E., (coord.), D. Block, A. Candela, I. Fuenlabrada, L. Navarro, E. Taboada, et. al, Conafe-DIE, México, 1993. 